\title{
Rapid and Easy Extracellular Vesicle Detection on a Surface- Functionalized Power-Free Microchip toward Point-of-Care Diagnostics
}

\author{
Ryo Ishihara, ${ }^{*}, \dagger$ Tadaaki Nakajima, ${ }^{\ddagger}$ Yoshitaka Uchino, ${ }^{\dagger}$ Asuka Katagiri, ${ }^{\dagger}$ Kazuo Hosokawa, ${ }^{\S}$ \\ Mizuo Maeda, ${ }^{\S}$ Yasuhiro Tomooka, ${ }^{\ddagger}$ and Akihiko Kikuchi ${ }^{\dagger}$ \\ ${ }^{\dagger}$ Department of Materials Science and Technology and ${ }^{\ddagger}$ Department of Biological Science and Technology, Tokyo University of \\ Science, 6-3-1 Niijuku, Katsushika, Tokyo 125-8585, Japan \\ ${ }^{\S}$ Bioengineering Laboratory, RIKEN, 2-1 Hirosawa, Wako, Saitama 351-0198, Japan
}

Supporting Information

ABSTRACT: Extracellular vesicles (EVs) are promising novel cancer biomarkers. However, rapid and easy analysis of EVs is challenging because conventional detection methods require large sample volumes and long detection times. Microchipbased analytical systems have particularly attracted attention for development of point-of-care (POC) diagnostics. Previously, various biomarker detection methods on a portable power-free poly(dimethylsiloxane) (PDMS) microchip using laminar flow-assisted dendritic amplification have been developed. Recently, for easy functionalization, we proposed a microchannel inner surface-functionalized power-free PDMS microchip (SF-PF microchip) utilizing electron beam-induced graft polymerization. In this study, we apply the technique and prepare a novel SF-PF microchip. On the microchip, EVs were successfully detected. The required sample volume was $1.0 \mu \mathrm{L}$, and the total analysis time was 20 min. The microchip can contribute to EV-based POC cancer diagnosis.

\section{INTRODUCTION}

Early disease detection plays a vital role in effective treatment. To detect diseases via minimally invasive procedures, various biomarkers in liquid biopsies have been utilized. In the case of cancer, biomolecules such as proteins, ${ }^{1,2}$ circulating cell-free DNAs, ${ }^{3}$ and microRNAs ${ }^{4}$ have been reported as biomarkers. In many biomarkers, extracellular vesicles (EVs) have emerged as promising novel biomarkers of diseases, especially in pancreatic cancer, because of their high sensitivity and specificity of detection. $^{5}$

EVs, including exosomes and microvesicles, are small membranous particles originating from cells. Some EVs such as exosomes ${ }^{6,7}$ are secreted from inside the cell by exocytosis, and others such as extosomes ${ }^{8}$ and oncosomes, ${ }^{9}$ which are categorized as microvesicles, are directly budded from the cell membrane. They exist in body fluids such as saliva, blood, and urine. One of the roles of EVs in biological mechanisms as a cell-to-cell communication tool was revealed in $2007,{ }^{10}$ and EVs have since then attracted much attention. Roles of the EVs have been investigated for cancer, ${ }^{11}$ immune response, ${ }^{7}$ and development. ${ }^{12}$ As for cancers, reciprocal signals between cancer cells and the surrounding cells mediated by EVs are involved in cancer cell survival, metastasis, ${ }^{9,13}$ and the promotion of invasion, angiogenesis, and drug resistance. ${ }^{11,14}$ Because EVs carry molecular information about the origin cells, ${ }^{15,16}$ capturing this information could be useful in clinical

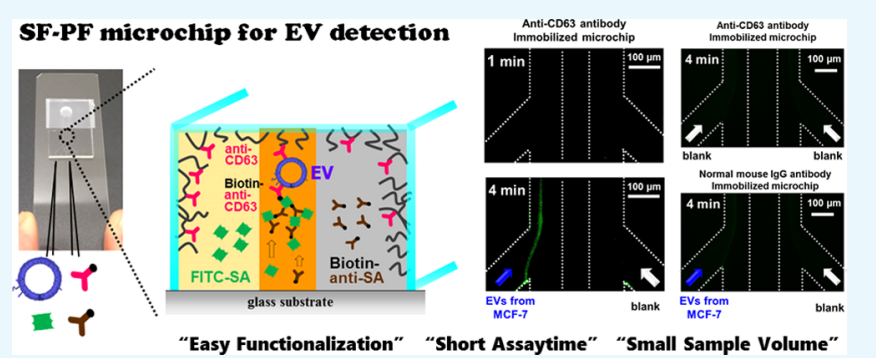

"Easy Functionalization" "Short Assaytime" "Small Sample Volume" cancer research. In addition to the existence of cancer-specific EVs, ${ }^{5}$ the size of the EVs could be information. ${ }^{17}$ Furthermore, the diagnostic biomarker potential of EVs has also been reported not only in cancers but also in diseases such as hepatitis $\mathrm{C},{ }^{18}$ multiple sclerosis, ${ }^{19}$ and malaria. ${ }^{20}$ Currently, EVs are expected to be a promising low-invasive biomarker.

However, rapid and easy analysis of EVs is challenging, and hence conventional detection methods, such as ultracentrifugation, western blotting, and enzyme-linked immunosorbent assays, require large sample volumes and long detection times. ${ }^{15}$ At the laboratory level, several EV detection methods have been reported, ${ }^{21-23}$ for example, ExoScreen, which is a bead-based EV detection method, requires $5 \mu \mathrm{L}$ of sample and $1.5 \mathrm{~h}^{24}$ To achieve the early detection of cancer, point-of-care (POC) diagnosis is a promising method. For POC diagnosis, microchip-based analytical systems have attracted attention because of their cost-effectiveness, that is, small sample sizes with short analysis times. Various biomarker detection methods on a power-free poly(dimethylsiloxane) (PDMS) microchip ${ }^{25}$ using laminar flow-assisted dendritic amplification (LFDA) ${ }^{26}$ have been developed ${ }^{27,28}$ by Hosokawa et al. The power-free sequential injection was based on the high gas solubility of

Received: August 7, 2017

Accepted: September 29, 2017

Published: October 12, 2017 
PDMS. Briefly, after PDMS is degassed in a vacuum chamber, air redissolves into PDMS at atmospheric pressure. The redissolution of the air in the closed microchannels provides negative pressure and enables power-free sample injection into the microchannels. In addition, the sequential injection is achievable because the first solution retains by capillary force at the inlet and second and third solutions restart the following injection without invasion of bubbles in the microchannels. The power-free technique eliminates the need for an external power source to supply the sample solution into the microchannels and makes the microchip portable.

Recently, we proposed a surface-functionalized power-free PDMS microchip (SF-PF microchip) ${ }^{29}$ as a novel platform for analysis, in which the inner surface of the microchip was chemically modified by electron beam-induced graft polymerization (EIGP). The portable SF-PF microchip enables rapid analyte detection from small sample volume. We selected EIGP in various surface modification methods of PDMS microchannels ${ }^{30}$ because it provides stable covalent modification and easily functionalizable surfaces. In our previous study, we confirmed that poly(glycidyl methacrylate) (PGMA)-grafted PDMS could be preserved for over 1 year and the power-free ability was retained even after surface functionalization. ${ }^{29}$ As the microchip can be easily functionalized with appropriate ligands at desired times, it can also be used for various applications. In this study, we immobilized antibodies on the inner surface of the microchannel to capture EVs and prepared a novel SF-PF microchip that enables rapid and easy EV detection. The proposed SF-PF microchip-based EV detection method would contribute to development of $\mathrm{EV}$ research and establishment of novel POC diagnosis.

\section{RESULTS AND DISCUSSION}

Characterization of the Prepared Microchip and Isolated EVs. For glycidyl methacrylate (GMA) polymerization, a dose of $20 \mathrm{kGy}$ was selected for reducing autofluorescence noise during EV detection and 3.0 M GMA was used in the feed for grafting to obtain the maximum amount of grafted PGMA on the inner surface of the microchannels on the microchip, as discussed in detail elsewhere. ${ }^{29}$ Immobilization of anti-CD63 antibody on the inner surface of the microchannels was confirmed by adsorption of fluorescently labeled secondary antibodies (Figure 1). The size distribution of isolated EVs from the supernatant of MCF7, which was obtained by NanoSight, is shown in Figure 2. As the size of the isolated EVs was approximately $30-100 \mathrm{~nm}$, the nanoparticles were categorized $^{31}$ as exosomes, ${ }^{6}$ which are secreted from inside the cell by exocytosis, or ectosomes, ${ }^{8}$ which are directly budded from the cell membrane. The concentration was $4.6 \times 10^{9} \mathrm{EVs} / \mu \mathrm{L}$ PBS.
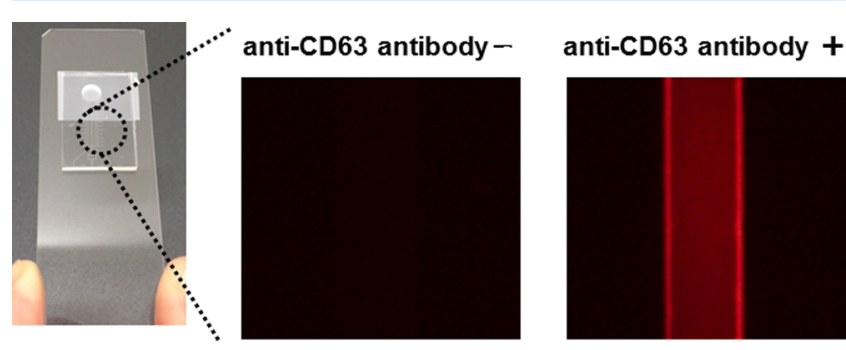

Figure 1. Anti-human CD63 antibody immobilization on the inner surface of the SF-PF microchip.

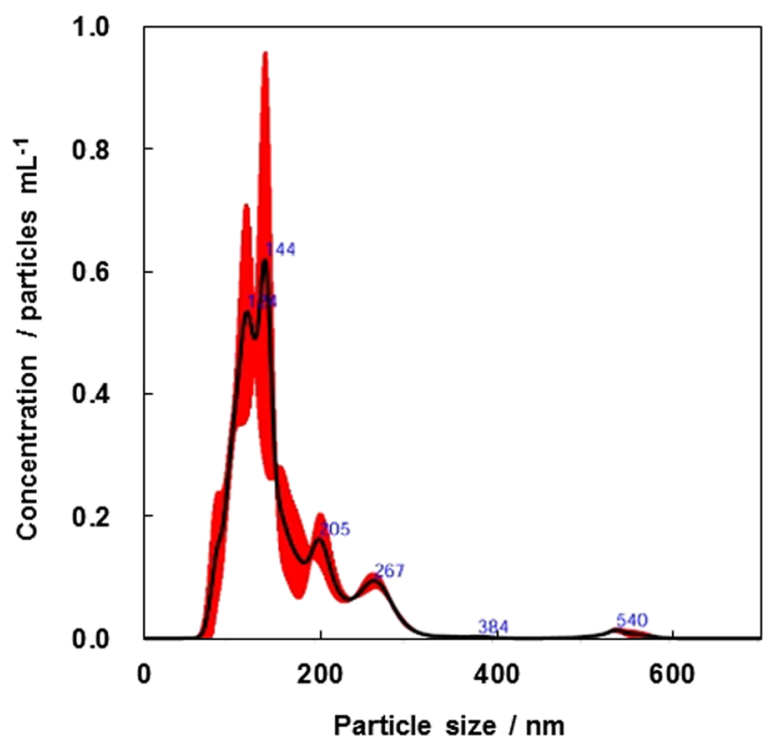

Figure 2. Size distribution of isolated EVs from conditioned medium of MCF7 cells.

EV Detection on the SF-PF Microchip. Typical fluorescent images of EV detection on the SF-PF microchip are shown in Figure 3a. On the anti-CD63 antibodyimmobilized microchip, with increasing amplification time, a bright line appeared only on the border between the amplification reagents in the left microchannel in which the target EV-containing solution $\left(4.6 \times 10^{9} \mathrm{EVs} / \mu \mathrm{L} \mathrm{PBS}\right)$ had been injected. On the other hand, the bright line did not appear even after $4 \mathrm{~min}$ in the microchannels in which blank solutions had been injected from both inlets. On the normal mouse IgGimmobilized microchip instead of that immobilized with antiCD63 antibody, the bright line also did not appear after 4 min in the microchannels in which the EV-containing solution was injected from the left inlet and blank solution was injected from the right inlet. The statistical evaluations of the fluorescent signals are shown in Figure $3 \mathrm{~b}$. The differences in the signal-toblank ratio between EV-blank and blank-blank on the antiCD63 antibody-immobilized microchip and between EV-blank on the anti-CD63 antibody-immobilized microchip and EVblank on the normal mouse IgG-immobilized microchip were statistically significant at $p<0.05$ and 0.01 , respectively. The differences in the signal-to-blank ratios between EV-blank on the normal mouse IgG-immobilized microchip and blankblank on both anti-CD63 antibody-immobilized and normal mouse IgG-immobilized microchips were not statistically significant. In addition, the differences in the signal-to-blank ratios between EV-blank and blank-blank on anti-CD63 antibody-impregnated bare PDMS microchips were not statistically significant (Figure S1). The EV concentration detected in this study was between 10 and 100 times higher than that in body fluids. ${ }^{32}$ Therefore, further study of the sensitivity improvement is needed prior to use in practical applications. The required sample volume was $1.0 \mu \mathrm{L}$, and the total analysis time was $20 \mathrm{~min}$. In conclusion, EVs were easily, rapidly, and specifically detected on the portable SF-PF microchip.

\section{CONCLUSIONS}

EVs are promising cancer biomarkers because they carry molecular information of the origin cells. In this study, we 
(a)
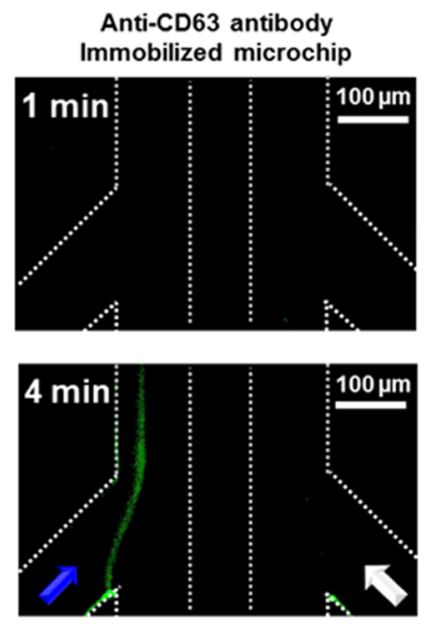

EVs from MCF-7
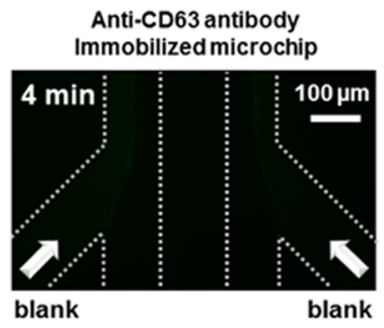

Normal mouse IgG antibody Immobilized microchip

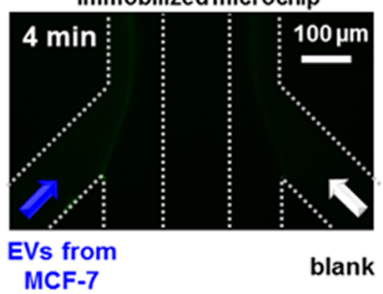

(b)

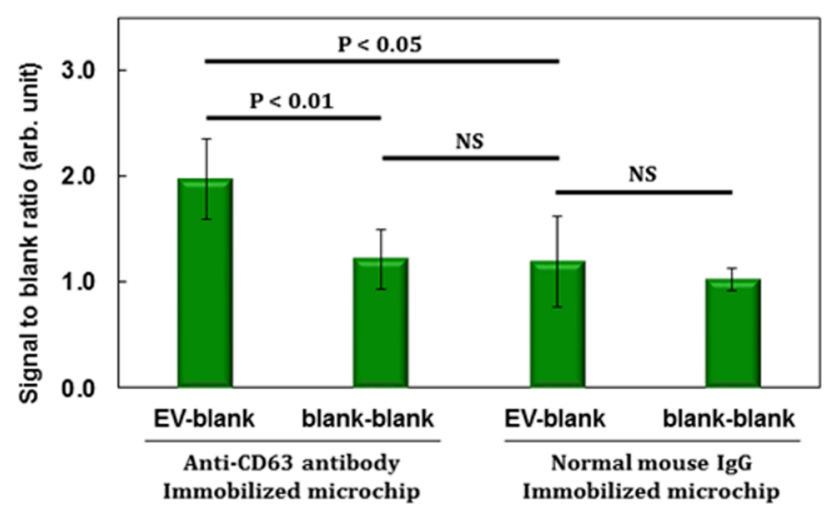

Figure 3. EV detection on the SF-PF microchip. (a) Typical fluorescent images and (b) statistical evaluation of the specific EV detection on the SF-PF microchip. EV-blank: EVs containing phosphate-buffered saline (PBS) injection from the left inlet and PBS injection from the right inlet.

proposed a simple inner surface modification method utilizing EIGP and applied the method to a power-free microchip. The SF-PF microchip demonstrated selective EV detection from a $1.0 \mu \mathrm{L}$ sample solution within $20 \mathrm{~min}$. Because previous EV detection methods take more than several hours and require more complicated operations, the SF-PF microchip-based rapid and easy EV detection method is superior. Although the SF-PF microchip is promising for EV-based diagnosis, improvements in sensitivity are required for practical applications. Protocol

optimization, the rational design of the microchannels to enhance the contact efficiency between the target EVs and the functionalized microchannel inner surfaces, and partial grafting $^{33}$ to avoid the consumption of EVs are possible solutions. Further study is urgently needed, which would contribute to a better understanding of EVs in biology and help to establish POC cancer diagnosis.

\section{MATERIALS AND METHODS}

Preparation of a Surface-Functionalized Power-Free (SF-PF) Microchip for EV Detection. The SF-PF microchip was prepared as follows. First, PDMS (Sylgard 184; Dow Corning, Midland, MI) with a pair of Y-shaped microchannels (100 $\mu \mathrm{m}$ in width, $25 \mu \mathrm{m}$ in height) was fabricated by soft lithography, as described previously. ${ }^{34}$ The left and right Yshaped microchannels are for the detection of the target EV and a blank reference, respectively. Then, PGMA was grafted onto the inner surface of the microchannels by EIGP (Figure 4), as described previously. ${ }^{29}$ Briefly, the microchannels of the PDMS microchip were filled with 3.0 M GMA monomer (Wako Pure Chemical Industries, Japan) in N,N-dimethylformamide solution and the microchip was set in a gas-barrier bag (Lamizip; AS ONE Corp., Japan) with an oxygen scavenger (Ageless; Mitsubishi Gas Chemical Co., Japan). The microchip was then irradiated with a $20 \mathrm{kGy}$ electron beam. After washing with methanol, a PGMA-grafted PDMS microchip was obtained. Next, human anti-CD63 antibody was immobilized onto the inner surface of the grafted microchip. Microchannels were filled with $1 \mathrm{mg} / \mathrm{L}$ anti-CD63 antibody (Sigma, St. Louis, $\mathrm{MO}$ ) in carbonate buffer solution and incubated at $310 \mathrm{~K}$ for 2 h. The amount of anti-CD63 antibody immobilized was evaluated using the fluorescently labeled secondary antibody (Alexa Fluor 594 hydrazide; Thermo Fisher Scientific, Waltham, MA). Finally, the antibody-immobilized PDMS microchip was degassed at $10 \mathrm{kPa}$ for $1 \mathrm{~h}$ and a SF-PF microchip for EV detection was obtained.

EV Isolation. According to a standard protocol for the isolation of $\mathrm{EVs},{ }^{35} \mathrm{EVs}$ were purified from conditioned medium of the breast cancer cell line, MCF7, by differential ultracentrifugation. MCF7 cells were cultured in $10 \mathrm{~mL}$ of RPMI1640 (Wako) supplemented with $10 \%$ fetal bovine serum (Cansera International, Inc., Ontario, Canada), nonessential amino acids (Wako), penicillin $(31 \mu \mathrm{g} / \mathrm{mL}$, Sigma), and streptomycin $(50 \mu \mathrm{g} / \mathrm{mL}$, Sigma $)$ in $100 \mathrm{~mm}$ dishes. The MCF7 cells $\left(3 \times 10^{6}\right)$ were passaged into eight dishes and cultured for 1 week. The supernatant $(80 \mathrm{~mL})$ was then harvested and centrifuged at $300 \mathrm{~g}$ at $277 \mathrm{~K}$ for $10 \mathrm{~min}$ to remove the cells, at $2000 \mathrm{~g}$ at $277 \mathrm{~K}$ for $20 \mathrm{~min}$ to remove the dead cells, and at $10000 \mathrm{~g}$ at $277 \mathrm{~K}$ for $45 \mathrm{~min}$ to remove cellular debris. The supernatant was ultracentrifuged to obtain an EV pellet at $110000 \mathrm{~g}$ at $277 \mathrm{~K}$ for $80 \mathrm{~min}$. The pellet was

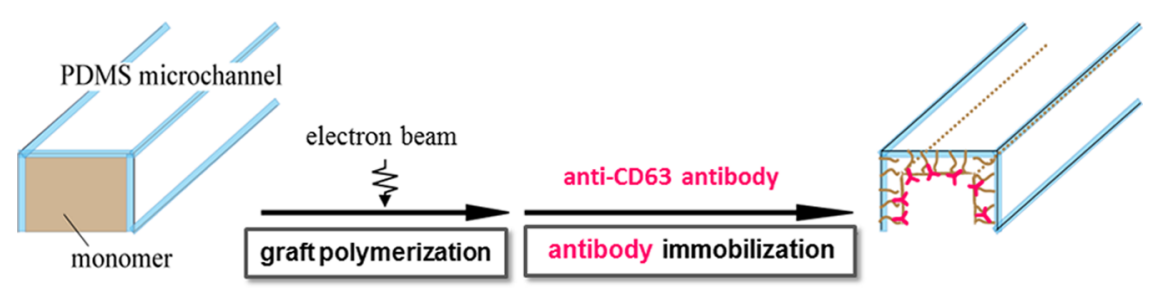

Figure 4. Scheme of the preparation of inner surface-functionalized power-free PDMS microchip (SF-PF microchip) for extracellular vesicle (EV) detection using electron beam-induced graft polymerization. 
suspended in phosphate-buffered saline (PBS) and ultracentrifuged again. The pellet was resuspended in approximately $200 \mu \mathrm{L}$ of PBS. The size distribution and concentration of the EVs were determined by NanoSight (NanoSight, Ltd., Wiltshire, SP4 7RT, U.K.) nanoparticle tracking analysis.

EV Detection. The isolated EVs were detected on the SFPF microchip. A schematic and protocol of EV detection are shown in Figures 5 and S2. Blocking buffer (1\% Roche

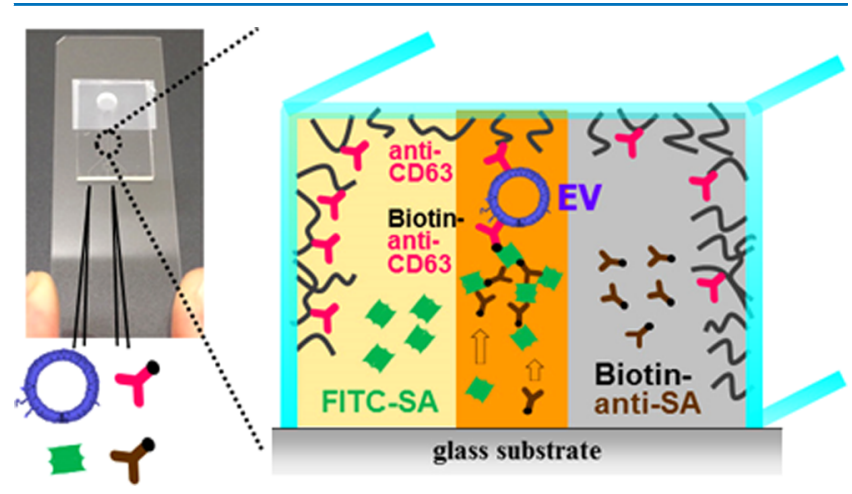

Figure 5. EV detection method: laminar flow-assisted dendritic amplification. FITC-SA: fluorescein isothiocyanate-conjugated streptavidin, biotin-anti-SA: biotinylated anti-streptavidin, anti-CD63: antihuman CD63 antibody, biotin-anti-CD63: biotinylated anti-human CD63 antibody.

Blocking Reagent, $0.02 \% \mathrm{w} / \mathrm{v}$ sodium dodecyl sulfate, $5 \times$ saline sodium citrate, and $0.05 \%$ Tween 20, Roche, Basel, Switzerland) was injected into the microchannel for $3 \mathrm{~min}$. Subsequently, $1.0 \mu \mathrm{L}$ of the target EV-containing PBS solution and $1.0 \mu \mathrm{L}$ of biotinylated $10 \mathrm{nM}$ anti-CD63 antibody in PBS were injected into the microchannel for $5 \mathrm{~min}$ to obtain the antibody-EV-antibody complex. Finally, the fluorescence signal was amplified by LFDA. ${ }^{26}$ Two amplification reagents, fluorescein isothiocyanate-conjugated streptavidin ("FITC-SA", $5.0 \mu \mathrm{g} / \mathrm{mL}$, Figure 5) and biotinylated anti-streptavidin ("Biotin-anti-SA", $20 \mu \mathrm{g} / \mathrm{mL}$, Figure 5), were injected into the microchannel. The solutions were injected into the grafted microfluidic device by power-free sequential injection, as described in detail elsewhere. ${ }^{36}$ The fluorescence intensities were quantified using the ImageJ software (version 1.45s, National Institute of Health, MD). The specificity of the SF-PF microchip to EVs was confirmed using normal mouse IgG (Santa Cruz Biotechnology, Santa Cruz, CA)-immobilized PF microchip instead of anti-CD63 antibody. The necessity of the graft polymer on the inner surface of the microchip was confirmed using an anti-CD63 antibody-impregnated bare PDMS microchip. The statistical significance of fluorescent signal intensities was assessed by the two-tailed $t$ test.

\section{ASSOCIATED CONTENT}

\section{S Supporting Information}

The Supporting Information is available free of charge on the ACS Publications website at DOI: 10.1021/acsomega.7b01147.

EV detection on an anti-CD63 antibody-immobilized bare PDMS microchip, detailed EV detection protocol by laminar flow-assisted dendritic amplification on the SFPF microchip (PDF)

\section{AUTHOR INFORMATION}

\section{Corresponding Author}

*E-mail: r.ishihara@rs.tus.ac.jp. Tel: +81-3-5876-1415. Fax: +81-3-5876-1639.

ORCID 1

Ryo Ishihara: 0000-0003-4862-4843

Notes

The authors declare no competing financial interest.

\section{ACKNOWLEDGMENTS}

This work was partly supported by JSPS KAKENHI grant numbers 25871118 and 16K12901.

\section{REFERENCES}

(1) Lilja, H.; Ulmert, D.; Vickers, A. J. Prostate-specific antigen and prostate cancer: prediction, detection and monitoring. Nat. Rev. Cancer 2008, 8, 268-278.

(2) Polanski, M.; Anderson, N. L. A List of Candidate Cancer Biomarkers for Targeted Proteomics. Biomarkers Insights 2006, 1, 148.

(3) Gormally, E.; Caboux, E.; Vineis, P.; Hainaut, P. Circulating free DNA in plasma or serum as biomarker of carcinogenesis: Practical aspects and biological significance. Mutat. Res., Rev. Mutat. Res. 2007, 635, 105-117.

(4) Mitchell, P. S.; Parkin, R. K.; Kroh, E. M.; Fritz, B. R.; Wyman, S. K.; Pogosova-Agadjanyan, E. L.; Peterson, A.; Noteboom, J.; O’Briant, K. C.; Allen, A.; Lin, D. W.; Urban, N.; Drescher, C. W.; Knudsen, B. S.; Stirewalt, D. L.; Gentleman, R.; Vessella, R. L.; Nelson, P. S.; Martin, D. B.; Tewari, M. Circulating microRNAs as stable bloodbased markers for cancer detection. Proc. Natl. Acad. Sci. U.S.A. 2008, 105, 10513-10518.

(5) Melo, S. A.; Luecke, L. B.; Kahlert, C.; Fernandez, A. F.; Gammon, S. T.; Kaye, J.; LeBleu, V. S.; Mittendorf, E. A.; Weitz, J.; Rahbari, N.; Reissfelder, C.; Pilarsky, C.; Fraga, M. F.; Piwnica-Worms, D.; Kalluri, R. Glypican-1 identifies cancer exosomes and detects early pancreatic cancer. Nature 2015, 523, 177-182.

(6) Théry, C. Exosomes: secreted vesicles and intercellular communications. F1000 Biol. Rep. 2011, 3, 15.

(7) Théry, C.; Ostrowski, M.; Segura, E. Membrane vesicles as conveyors of immune responses. Nat. Rev. Immunol. 2009, 9, 581593.

(8) Cocucci, E.; Meldolesi, J. Ectosomes and exosomes: shedding the confusion between extracellular vesicles. Trends Cell Biol. 2015, 25, 364-372.

(9) Di Vizio, D.; Kim, J.; Hager, M. H.; Morello, M.; Yang, W.; Lafargue, C. J.; True, L.; Rubin, M. A.; Adam, R. M.; Beroukhim, R; Demichelis, F.; Freeman, M. R. Oncosome formation in prostate cancer: Association with a region of frequent chromosomal deletion in metastatic disease. Cancer Res. 2009, 69, 5601-5609.

(10) Valadi, H.; Ekstrom, K.; Bossios, A.; Sjostrand, M.; Lee, J. J.; Lotvall, J. O. Exosome-mediated transfer of mRNAs and microRNAs is a novel mechanism of genetic exchange between cells. Nat. Cell Biol. 2007, 9, 654-659.

(11) Wendler, F.; Favicchio, R.; Simon, T.; Alifrangis, C.; Stebbing, J.; Giamas, G. Extracellular vesicles swarm the cancer microenvironment: from tumor-stroma communication to drug intervention. Oncogene 2016, 36, 877-884.

(12) Nakano, S.; Yamamoto, S.; Okada, A.; Nakajima, T.; Sato, M.; Takagi, T.; Tomooka, Y. Role of extracellular vesicles in the interaction between epithelial and mesenchymal cells during oviductal ciliogenesis. Biochem. Biophys. Res. Commun. 2017, 483, 245-251.

(13) Hoshino, A.; Costa-Silva, B.; Shen, T.-L.; Rodrigues, G.; Hashimoto, A.; Tesic Mark, M.; Molina, H.; Kohsaka, S.; Di Giannatale, A.; Ceder, S.; Singh, S.; Williams, C.; Soplop, N.; Uryu, K.; Pharmer, L.; King, T.; Bojmar, L.; Davies, A. E.; Ararso, Y.; Zhang, T.; Zhang, H.; Hernandez, J.; Weiss, J. M.; Dumont-Cole, V. D.; Kramer, K.; Wexler, L. H.; Narendran, A.; Schwartz, G. K.; Healey, J. 
H.; Sandstrom, P.; Jørgen Labori, K.; Kure, E. H.; Grandgenett, P. M.; Hollingsworth, M. A.; de Sousa, M.; Kaur, S.; Jain, M.; Mallya, K.; Batra, S. K.; Jarnagin, W. R.; Brady, M. S.; Fodstad, O.; Muller, V.; Pantel, K.; Minn, A. J.; Bissell, M. J.; Garcia, B. A.; Kang, Y.; Rajasekhar, V. K.; Ghajar, C. M.; Matei, I.; Peinado, H.; Bromberg, J.; Lyden, D. Tumour exosome integrins determine organotropic metastasis. Nature 2015, 527, 329-335.

(14) Ciardiello, C.; Cavallini, L.; Spinelli, C.; Yang, J.; Reis-Sobreiro, M.; de Candia, P.; Minciacchi, V.; Di Vizio, D. Focus on Extracellular Vesicles: New Frontiers of Cell-to-Cell Communication in Cancer. Int. J. Mol. Sci. 2016, 17, 175.

(15) Yoshioka, Y.; Kosaka, N.; Konishi, Y.; Ohta, H.; Okamoto, H.; Sonoda, H.; Nonaka, R.; Yamamoto, H.; Ishii, H.; Mori, M.; Furuta, K.; Nakajima, T.; Hayashi, H.; Sugisaki, H.; Higashimoto, H.; Kato, T.; Takeshita, F.; Ochiya, T. Ultra-sensitive liquid biopsy of circulating extracellular vesicles using ExoScreen. Nat. Commun. 2014, 5, No. 3591.

(16) Im, H.; Shao, H.; Park, Y. I.; Peterson, V. M.; Castro, C. M.; Weissleder, R; Lee, H. Label-free detection and molecular profiling of exosomes with a nano-plasmonic sensor. Nat. Biotechnol. 2014, 32, 490-495.

(17) Sharma, S.; Gillespie, B. M.; Palanisamy, V.; Gimzewski, J. K. Quantitative Nanostructural and Single-Molecule Force Spectroscopy Biomolecular Analysis of Human-Saliva-Derived Exosomes. Langmuir 2011, 27, 14394-14400.

(18) Kornek, M.; Lynch, M.; Mehta, S. H.; Lai, M.; Exley, M.; Afdhal, N. H.; Schuppan, D. Circulating Microparticles as Disease-Specific Biomarkers of Severity of Inflammation in Patients With Hepatitis C or Nonalcoholic Steatohepatitis. Gastroenterology 2012, 143, 448-458.

(19) Sáenz-Cuesta, M.; Osorio-Querejeta, I.; Otaegui, D. Extracellular Vesicles in Multiple Sclerosis: What are They Telling Us? Front. Cell. Neurosci. 2014, 8, 100.

(20) Mfonkeu, J. B. P.; Gouado, I.; Kuaté, H. F.; Zambou, O.; Zollo, P. H. A.; Grau, G. E. R.; Combes, V. Elevated Cell-Specific Microparticles Are a Biological Marker for Cerebral Dysfunctions in Human Severe Malaria. PLoS One 2010, 5, No. e13415.

(21) Zhang, P.; He, M.; Zeng, Y. Ultrasensitive microfluidic analysis of circulating exosomes using a nanostructured graphene oxide/ polydopamine coating. Lab Chip 2016, 16, 3033-3042.

(22) Kanwar, S. S.; Dunlay, C. J.; Simeone, D. M.; Nagrath, S. Microfluidic device (ExoChip) for on-chip isolation, quantification and characterization of circulating exosomes. Lab Chip 2014, 14, 18911900.

(23) Zhao, Z.; Yang, Y.; Zeng, Y.; He, M. A microfluidic ExoSearch chip for multiplexed exosome detection towards blood-based ovarian cancer diagnosis. Lab Chip 2016, 16, 489-496.

(24) Yoshioka, Y.; Kosaka, N.; Konishi, Y.; Ohta, H.; Okamoto, H.; Sonoda, H.; Nonaka, R.; Yamamoto, H.; Ishii, H.; Mori, M.; Furuta, K.; Nakajima, T.; Hayashi, H.; Sugisaki, H.; Higashimoto, H.; Kato, T.; Takeshita, F.; Ochiya, T. Ultra-sensitive liquid biopsy of circulating extracellular vesicles using ExoScreen. Nat. Commun. 2014, 5, No. 3591.

(25) Hosokawa, K.; Sato, K.; Ichikawa, N.; Maeda, M. Power-free poly(dimethylsiloxane) microfluidic devices for gold nanoparticlebased DNA analysis. Lab Chip 2004, 4, 181-185.

(26) Hosokawa, K.; Omata, M.; Maeda, M. Immunoassay on a Power-Free Microchip with Laminar Flow-Assisted Dendritic Amplification. Anal. Chem. 2007, 79, 6000-6004.

(27) Okada, H.; Hosokawa, K.; Maeda, M. Power-Free Microchip Immunoassay of PSA in Human Serum for Point-of-Care Testing. Anal. Sci. 2011, 27, 237.

(28) Ishihara, R.; Hasegawa, K.; Hosokawa, K.; Maeda, M. Multiplex MicroRNA Detection on a Power-free Microfluidic Chip with Laminar Flow-assisted Dendritic Amplification. Anal. Sci. 2015, 31, 573-576.

(29) Ishihara, R.; Uchino, Y.; Hosokawa, K.; Maeda, M.; Kikuchi, A. Preparation of a Surface-functionalized Power-free PDMS Microchip for MicroRNA Detection Utilizing Electron Beam-induced Graft Polymerization. Anal. Sci. 2017, 33, 197-202.
(30) Makamba, H.; Kim, J. H.; Lim, K.; Park, N.; Hahn, J. H. Surface modification of poly(dimethylsiloxane) microchannels. Electrophoresis 2003, 24, 3607-3619.

(31) van der Pol, E.; Böing, A. N.; Harrison, P.; Sturk, A.; Nieuwland, R. Classification, Functions, and Clinical Relevance of Extracellular Vesicles. Pharmacol. Rev. 2012, 64, 676-705.

(32) Eitan, E.; Green, J.; Bodogai, M.; Mode, N. A.; Bæk, R.; Jørgensen, M. M.; Freeman, D. W.; Witwer, K. W.; Zonderman, A. B.; Biragyn, A.; Mattson, M. P.; Hooten, N. N.; Evans, M. K. Age-Related Changes in Plasma Extracellular Vesicle Characteristics and Internalization by Leukocytes. Sci. Rep. 2017, 7, No. 1342.

(33) Arata, H.; Komatsu, H.; Hosokawa, K.; Maeda, M. Rapid and sensitive microRNA detection with laminar flow-assisted dendritic amplification on power-free microfluidic chip. PLoS One 2012, 7 , No. e48329.

(34) Hosokawa, K.; Fujii, T.; Endo, I. Handling of Picoliter Liquid Samples in a Poly(dimethylsiloxane)-Based Microfluidic Device. Anal. Chem. 1999, 71, 4781-4785.

(35) Thery, C.; Amigorena, S.; Raposo, G.; Clayton, A. Current Protocols in Cell Biology; John Wiley \& Sons, Inc.: New York, 2006.

(36) Hosokawa, K.; Omata, M.; Sato, K.; Maeda, M. Power-free sequential injection for microchip immunoassay toward point-of-care testing. Lab Chip 2006, 6, 236-241. 